\title{
Impacto de las acciones de un programa de enfermería con enfoque promocional y de autocuidado en la capacidad funcional de adultos mayores
}

\author{
Impact of the actions of a nursing program with a promotional approach and self-care in the functional capacity \\ of older adults
}

Elsy Cecilia Puello-Alcocer ${ }^{*}$ orcid.org/0000-0003-0914-1857

Concepción Amador-Ahumada'orcid.org/0000-0002-0008-3374

Jorge Eliecer Ortega-Montes² orcid.org/0000-0003-4605-6061

1 Programa de Enfermería, Facultad de Ciencias de la Salud. Universidad de Córdoba. Montería, Colombia

2 Programa de Ciencias Sociales, Facultad de Educación y Ciencias Humanas. Universidad de Córdoba. Montería, Colombia

Fecha de recepción: Septiembre 15 - 2016 Fecha de revisión: Noviembre 15 - 2016 Fecha de aceptación: Agosto 11 - 2017

Puello-Alcocer EC, Amador-Ahumada C, Ortega-Montes JE. Impacto de las acciones de un programa de enfermería con enfoque promocional y de autocuidado en la capacidad funcional de adultos mayores. Univ. Salud. 2017;19(2):152-162. DOI: http://dx.doi.org/10.22267/rus.171902.78

\section{Resumen}

Introducción: Los aportes de los profesionales de la salud apoyados en el modelo de Promoción permiten evidenciar actitudes, motivaciones y sentimientos del adulto mayor. Objetivo: Analizar el impacto de las acciones de un Programa de Enfermería con enfoque promocional y de autocuidado, en el fortalecimiento de la capacidad funcional en adultos mayores. Materiales y métodos: Estudio descriptivo-prospectivo y de intervención pre y posttest sin grupo control en 75 personas; muestreo por conveniencia. Se realizó recolección de datos mediante encuesta y Escala de Barthel. Resultados: El 48\% de la población estaba en el rango etario entre 67 y 72 años, 74\% sexo femenino, $40 \%$ viudos y $57 \%$ analfabetas. Con los resultados del post-test se identificó una variación importante en la modificación de la categoría: dependencia moderada a dependencia leve $(71,43 \%)$ y dependencia leve a independencia (30,76\%); el 82\% desarrolló acciones de autocuidado, 80\% se mantuvo activo, 78\% mostró adherencia al tratamiento de enfermedades, 90\% mantiene higiene corporal. Conclusiones: Las acciones continuas de enfermería con enfoque promocional y de autocuidado permiten revertir limitaciones en condiciones funcionales de dependencia moderada a leve y de leve a independiente; conservan o potencializan la capacidad de regular su autosuficiencia y autonomía, beneficiando así la salud de adultos mayores.

Palabras clave: Adulto mayor; autonomía personal; autocuidado; Enfermería. (Fuente: DeCS, Bireme).

\begin{abstract}
Introduction: The contributions of health professionals supported by the promotion model allow to demonstrate attitudes, motivations and feelings of the elderly. Objective: To analyze the impact of the actions of a nursing program with a promotional and self-care approach in the strengthening of functional capacity in older adults. Materials and methods: Descriptive-prospective study and pre-and post-test intervention without control group in 75 people was carried out with a sampling for convenience. Data collection through survey and Barthel scale was conducted. Results: $48 \%$ of the population was in the age range between 67 and $72,74 \%$ were female, $40 \%$ were widowed people and $57 \%$ were illiterate. An important variation was identified in the modification of the category taking into account the results of the post-test: moderate dependence on mild dependence $(71.43 \%)$ and mild dependence on independence (30.76\%). 82\% developed self-care actions, $80 \%$ remained active, $78 \%$ showed adherence to disease treatment and $90 \%$ maintain body hygiene. Conclusions: Continuous nursing actions with a promotional and self-care approach allow reverting limitations in functional conditions of moderate to mild and
\end{abstract}


mild-to-independent dependence, they retain or potentiate the ability to regulate their self-sufficiency and autonomy, thus benefiting the health of older adults.

Key words: elderly, functionality, self-care, nursing. (Source: DeCS, Bireme).

\section{Introducción}

El proceso de salud enfermedad de las personas de edad avanzada debe abordarse con políticas públicas que conciban la prestación de un servicio con trato humanizado y pertinente, que responda a las particularidades y expectativas de las personas. Existe la necesidad de reemplazar las miradas tradicionalistas que colocan el acento en los altos costos de atención del adulto, por aquellas que motivan la adopción de concepciones integrales, donde los egresos económicos derivados de la atención en salud se asimilen como una inversión al mejoramiento de la funcionalidad y la autonomía física del sujeto contribuyendo a la calidad de vida de los $\operatorname{mismos}^{(1)}$.

Para la Organización Mundial de la Salud -OMS(2), el proceso de envejecimiento de la población mundial se produce a pasos acelerados, estimándose que entre 2015 y 2020 los adultos mayores con más de 60 años pasarán de 900 millones a 2000 millones, lo equivalente a decir que se tendrá un aumento demográfico en este segmento poblacional de $10 \%$ aproximadamente. Los avances en los países desarrollados en materia de atención en salud han sido evidentes, generando impactos directamente asociados con la disminución de los niveles de dependencia para la realización de las actividades cotidianas de los adultos mayores, sin embargo, prevalecen las limitaciones menos graves en materia de funcionalidad, y en lo que respecta a los países de ingresos bajos y medianos se puede evidenciar que la carga de morbilidad es mayor que en los países ricos(1).

En Colombia, la cifra de adultos mayores (60 y más años de edad) obedece a 4.962.491 equivalente al $10,53 \%$ de la población total, lo que refleja un importante incremento con relación al Censo de 2005. El análisis de la transición demográfica en el territorio nacional señala que el proceso de envejecimiento en grupos etarios mayores de 60 años presenta una tasa de crecimiento superior al de la población total; además, el índice de envejecimiento calculado como el peso de la población mayor de edad con relación a la población infantil y adolescente se triplicó, observándose una conducta ascendente en las últimas décadas pasando de 10 a 34 por cada 100 personas menores de 15 años entre 1964 - 2010(3). El departamento de Córdoba no se aleja de la tendencia nacional, las cifras que reporta el "Plan de Desarrollo 2016-2019”(4) estiman que 8.38\% de la población está ubicada en este rango poblacional equivalente a 128.606 adultos mayores de 60 años siendo uno de los departamentos que más crecimiento $(38,8 \%)$ reportará para el año 2020.

En el departamento de Córdoba se presentan características específicas para la prestación del servicio de salud, porque registra uno de los índices de pobreza multidimensional más alto de la región $(79,6 \%)$, indicador que se acentúa cuando se refiere a la zona rural $(90,1 \%)$ lo que equivale a 10 puntos porcentuales $(80,1 \%)$ por encima de la media nacional, y en lo que respecta al grado de desigualdad en la distribución del ingreso se obtuvo que el coeficiente de Gini para el año 2015 registró un 0,465 frente al 0,522 del nivel nacional(5).

Estas cifras analizadas bajo la óptica de las tendencias mundiales, indican una relación inversamente proporcional; a menor ingreso, mayor es la carga de morbilidad del adulto mayor, que indudablemente demanda una mayor responsabilidad del Estado y de otras instituciones para contrarrestar esta problemática. Lo anterior, justifica algunas propuestas gubernamentales como el Plan Decenal de Salud Pública -PDSP- 2012-2021(6) que incorpora enfoques y modelos de salud que orienta la atención y el cuidado desde una perspectiva de género y ciclo de vida desde los derechos humanos, partiendo de la idea de que 
las personas no pueden desligarse de los contextos socio-culturales donde se desarrollan, haciéndose necesario que el sistema de salud identifique los patrones y especificidades para que se brinde la atención integral y se propenda por el logro de los objetivos propuestos en los diferentes planes de desarrollo de salud en cada una de las regiones colombianas.

Son pertinentes los aportes del Modelo de Promoción de la Salud (MPS)(7), al enfatizar que las características individuales, las experiencias, conocimientos previos y afectos particulares de la conducta son vitales para propiciar estados motivacionales en el sujeto, que los aboque a participar activamente en el cuidado de su salud. A partir de la implementación de este modelo, el profesional de enfermería estará en la capacidad de comprender y potenciar las actitudes, motivaciones y sentimientos del adulto mayor a con base en el concepto de autoeficacia; valorando qué tanto responden las intervenciones de enfermería a las necesidades de cuidado del geronte.

Bajo estos requerimientos surge la necesidad de documentar los efectos de las acciones de cuidado de enfermería en el contexto familiar y comunitario en que se desenvuelven los adultos mayores, por ello, se adelantó el proceso investigativo para medir el efecto de las actividades de enfermería en la funcionalidad y en la salud de los adultos mayores sujetos del cuidado. Al evaluar los efectos de las acciones realizadas dentro del Programa de Enfermería los autores evidenciaron el fortalecimiento de la capacidad funcional y de autocuidado de los adultos mayores, que motivó la sistematización y publicación de los resultados de la experiencia con la población intervenida, debido a que estos hallazgos pueden servir como fundamento para la reorientación de la política pública y programas locales para la atención y cuidado de la salud de estos colectivos humanos.

\section{Materiales y métodos}

Estudio descriptivo, prospectivo y de intervención pre y post test sin grupo de control. Se desarrolló durante 18 meses, en el período comprendido desde febrero de 2014 hasta agosto de 2015.

El universo de este estudio fue de 150 adultos mayores, de los cuales se seleccionaron setenta y cinco (75) bajo un criterio de muestreo por conveniencia, dado que se hacía necesario trabajar con los adultos mayores adscritos al Programa de Extensión Solidaria de la Universidad de Córdoba, Colombia que se desarrolla en zonas circunvecinas a los predios de la sede central de la universidad, específicamente población de estrato socioeconómico 1, de los sectores Camilo Torres, 20 de julio y Villa Fátima, del barrio Mocarí, comuna 9 del Municipio de Montería. Los criterios de inclusión para participar en el proyecto fueron: a) ser mayor de 60 años; b) vivir en la comuna 9 del Municipio de Montería, c) estar vinculado al Programa de Extensión de Ancianos dirigido por los grupos de investigación Huellas Calidad de Vida y Desarrollo, Democracia y Educación de la Universidad de Córdoba; d) no poseer limitaciones físicas incapacitantes ni enfermedades mentales o neurológicas que impidan el proceso comunicativo y e) haber firmado el consentimiento informado.

Metodológicamente se utilizaron tres fases en el diseño, de manera que pudiese ser levantada la información para su sistematización; estas fueron:

Fase 1. Diagnóstico. En primera instancia se aplicó una encuesta y la Escala de Barthel( ${ }^{(8)}$ para determinar el nivel socio-sanitario y la funcionalidad del anciano, además, se obtuvo información relativa al autocuidado en Actividades de la Vida Diaria Básicas (AVDB).

Es importante anotar que la Escala de Barthel, evalúa la capacidad de autocuidado y funcionalidad en lo relativo a las AVDB, mediante la valoración de la habilidad funcional para realizar solo o con ayuda, 10 acciones correspondientes al cuidado personal como: capacidad para comer por sí solo, trasladarse entre la silla y la cama, aseo personal, uso del retrete, bañarse o ducharse, desplazarse, subir y bajar escaleras, vestirse y desvestirse, control de 
heces y control de orina; la ayuda es medida en diferentes gradaciones ( $<20$ a 100 puntos). La interpretación es la siguiente: Menos de 20 dependiente total; entre 20-35 dependiente grave; 40-55 dependiente moderado; mayor o igual a 60 dependiente leve y la obtención de 100 puntos indica una total independencia en las personas mayores(8).

Se realizaron actividades de valoración tales como tamizaje y control de tensión arterial, control de peso, medición de perímetro abdominal, control de glicemia (glucometría), tamizaje visual y aplicación de la escala de Barthel; sobre esa base diagnóstica se diseñaron diversas actividades de intervención y seguimiento.

Fase 2. Ejecución y seguimiento. Este momento correspondió al desarrollo de las acciones de enfermería, se realizaron actividades educativas, asistenciales, artísticas, físicas ocupacionales, recreativas, espirituales y de consejería; así mismo, ejercicios de motricidad fina, acciones de seguimiento y control de enfermedades crónicas identificadas en la fase diagnóstica.

Las acciones de promoción de la salud y prevención de la enfermedad se efectuaron mediante metodologías participativas e interactivas bajo los principios del Modelo de Promoción de la Salud (MSP) que señala tomar en consideración las particularidades y las experiencias significativas de las personas como estrategia de autoeficacia y motivación frente al autocuidado. Así mismo, la prevención de complicaciones e incapacidades generadas por las diferentes patologías presentes en los ancianos pertenecientes al programa; también, se les enfatizó que de los cuidados, estilos de vida y el buen manejo de su salud y su enfermedad dependía su funcionalidad, autonomía e independencia factores esenciales para un envejecimiento saludable y digno(7).

Las técnicas y el enfoque educativo empleado fueron personalizados $y$ acorde al nivel educativo y cultural de los adultos mayores, las acciones, los elementos y los escenarios fueron coherentes con los recursos requeridos y con la realidad del contexto en que participantes e investigadores estaban inmersos. La universidad puso a disposición el talento humano, recursos físicos y económicos para la ejecución. Los temas que se efectuaron en el proceso educativo fueron seleccionados con base en el análisis de la situación de salud encontrada, en las necesidades de auto cuidado identificadas en la primera fase $\mathrm{y}$ en las patologías sentidas por los adultos mayores que integraron la muestra.

Para generar una intervención más personalizada y asegurar al máximo comodidad y asistencia de los adultos mayores a los encuentros, se dividió la población sujeto en tres grupos acorde a su ubicación geográfica. Se realizaron 10 actividades en cada una de las sedes, de acuerdo a los objetivos de cada fase (uno y dos) para un total de 30 actividades con un promedio de 25 adultos mayores.

Finalizada esta etapa, se efectuó nuevamente valoración de las capacidades funcionales y de autocuidado en los participantes, este post test fue fundamental para realizar el análisis del impacto de las acciones solidarias del Programa de Enfermería en poblaciones de alta vulnerabilidad social.

Fase 3. Sistematización de hallazgos. Las observaciones se sistematizaron utilizando registros de campo e información estadística, considerando la necesidad de documentar diversos aspectos relacionados con evidencia del fortalecimiento de la capacidad funcional $\mathrm{y}$ autocuidado en los adultos mayores, confrontando los resultados previos $\mathrm{y}$ posteriores a la intervención de Enfermería, de dicha comparación fue posible extraer la información relativa al impacto de las acciones del programa.

Se organizó la información en gráficos, matrices de doble entrada, mapas conceptuales y cartografía socio sanitaria acorde a la naturaleza de los datos y dependiendo del contenido y enfoque de cada área temática. Los resultados se plasmaron en un informe completo y sus alcances de logros en términos de salud y bienestar han sido sujeto de discusiones 
académicas, de forma tal que se demuestre y se reconoce la importancia de la intervención del profesional de enfermería en las áreas gerontológica y geriátrica.

\section{Consideraciones éticas}

La postura ética se enmarcó en los lineamientos de la Resolución 8430 de 1993, inscribiéndose en los parámetros de una investigación sin riesgos la cual fue aprobada por el Comité de Ética en Investigación de la Facultad de Ciencias de la Salud de la Universidad de Córdoba; para garantía del cumplimiento de los aspectos éticos relativos a la participación voluntaria en propuestas de intervención se informó a los potenciales participantes, a sus familias $y$ cuidadores familiares principales sobre los objetivos, alcances, métodos y riesgos de las intervenciones a realizar; cada adulto mayor estuvo en condiciones de decidir libremente su intención de participar en el estudio, así como también estaban en libertad de retirarse cuando lo considerara conveniente.

Para controlar los riesgos éticos que podían surgir durante el desarrollo de las actividades físicas, toma de muestras, medición de las constantes vitales, ejercitación física y demás procedimientos requeridos en la valoración o en la intervención que ameritaban un contacto directo o riesgos potenciales a la integridad física o mental, se dispuso de un equipo de profesionales cualificados para intervenir las posibles eventualidades que se presentaran.

\section{Resultados}

\section{Perfil sociodemográfico}

Los participantes correspondieron a personas entre 62 y 91 años de edad. Los grupos de edad obedecieron a un $48 \%$ entre 67 y 72 años, $21 \%$ entre 73-77, un $20 \%$ se ubicó en el rango de 7882 años y las minorías obedecieron a los grupos de edad entre 62 a 66 años y 83 a 91 años.

En lo referente a la condición de género $74 \%$ eran mujeres; el estado civil de la muestra se distribuyó en $40 \%$ que refirió ser viudo, el $25 \%$ señaló la condición de unión libre, 22\% casado $8 \%$ solteros, y $5 \%$ separados. De tal forma que en la distribución 53\% de los adultos mayores carecen de apoyo de una pareja conyugal (Tabla $1)$.

Tabla 1. Perfil sociodemográfico de los sujetos en estudio

\begin{tabular}{lcc}
\hline & n & \% \\
\hline Edad & & \\
62 a 66 años & 5 & 5,33 \\
67 a 72 años & 46 & 48 \\
73 a 77 años & 16 & 21,33 \\
78 a 82 años & 15 & 20 \\
83 a 91 años & 4 & 5,33 \\
Género & & \\
Mujer & 56 & 74 \\
Hombre & 19 & 26 \\
Estado civil & & \\
Viudos & 30 & 40 \\
Unión libre & 18 & 25 \\
Casado & 16 & 22 \\
Soltero & 6 & 8 \\
Separado & 4 & 5 \\
Ingreso mensual: & \multicolumn{2}{c}{} \\
Ningún ingreso & 42 & 56 \\
Subsidio económico del Estado Colombiano & 29 & 39 \\
Menos de un salario mínimo; & 4 & 5 \\
Régimen de atención en salud: Subsidiado & 75 & 100 \\
Pensión: No recibe & 75 & 100 \\
Escolaridad: analfabeta & 43 & 57 \\
Complacido con su estilo de vida & 34 & 45 \\
Convivencia con familiares & 68 & 91 \\
\hline n= número de casos & &
\end{tabular}

$\mathrm{n}=$ número de casos

Más de la mitad los adultos mayores encuestados $(56 \%)$ no recibe ingresos mensuales, seguidos de $39 \%$ que manifestaron recibir subsidios del gobierno y $5 \%$ devenga menos de un salario mínimo legal vigente. Se destaca que el $100 \%$ recibe servicios de atención en salud en el régimen subsidiado $\mathrm{y}$ por sus condiciones ocupacionales de informalidad a lo largo de su vida, ninguno de los participantes tuvo la opción de poder acceder a pensión de jubilación.

En cuanto a las condiciones educativas el $57 \%$ de los sujetos de estudio declaró ser analfabeta y en lo que respecta a los factores psico-sociales $72 \%$ de los sujetos abordados percibe deterioro de su condición de salud, $45 \%$ se declara complacido con su estilo de vida, 91\% conviven con sus familiares en familias extensas y una minoría refieren vivir solos.

\section{Capacidad funcional}

Dentro de los hallazgos se pudo evidenciar que $49,3 \%$ de los adultos mayores son 
independientes en la realización de las actividades básicas cotidianas, seguidos de $48 \%$ que presentan dependencia leve y $2,7 \%$ dependencia moderada (Tabla 2).

Tabla 2. Calificación de la funcionalidad según la Escala Barthel antes y después

\begin{tabular}{lcccc}
\hline \multirow{2}{*}{ Criterios Escala Barthel } & \multicolumn{2}{c}{ Antes } & \multicolumn{2}{c}{ Después } \\
& n & \% & n & \% \\
\hline$<20$ dependiente total & - & - & - & - \\
20-35 dependiente grave & - & - & - & - \\
40-55 dependiente moderado & 7 & 9,3 & 2 & 2,7 \\
60 dependiente leve & 52 & 69,3 & 36 & 48,0 \\
100 independiente & 16 & 21,3 & 37 & 49,3 \\
\hline
\end{tabular}

De acuerdo con los resultados del post test, las acciones educativas, asistenciales, de consejería y actividades ocupacionales, lúdicas y físicas dirigidas, tuvieron un efecto positivo en la funcionalidad de los adultos mayores, en quienes se identificó una variación importante en la modificación de la categoría de dependencia moderada y leve que tenían al inicio. De tal forma que de siete participantes clasificados en dependencia moderada, dos (28,57\%) se mantuvieron en el mismo nivel en la Escala o Índice de Barthel (IB) y cinco evolucionaron a dependencia leve $(71,43 \%)$.

De los 52 participantes en dependencia leve, 16 $(30,76 \%)$ pasaron a la clasificación de independiente y 36 conservaron el mismo nivel en la escala $(69,24 \%)$. De manera que el número de adultos mayores independientes se duplicó al final de las intervenciones. Luego de efectuar la totalidad de las actividades, se identificó que los mejores puntajes obtenidos por los adultos mayores en el IB fueron: arreglarse $(99,4 \%)$ y lavarse $(99,2 \%)$; uso del retrete $(99 \%)$, control de esfínteres $(96,5 \%)$ y capacidad de vestirse $(95,8 \%)$.

Finalizadas las intervenciones, al examinar algunas de las AVDB se pudo identificar que aquellas con mayor grado de dificultad para obtener resultados provechosos esperados fueron la capacidad para subir y bajar escalones, no obstante, al finalizar $55 \%$ de los ancianos pudieron efectuar de forma independiente dicha función; lograr la continencia urinaria normal $(88,1 \%)$ y caminar sin ayuda hasta 50 metros $(88 \%)$.

Impacto de las intervenciones de enfermería en la salud de los ancianos. Concluidas las acciones de intervención y observaciones anecdóticas del proceso, se procedió a categorizar los niveles de impacto de las acciones realizadas en los 18 meses y se pudo identificar el alcance de las mismas en dos grandes aspectos de logros, los cuales se detallan a continuación:

\section{a) Fortalecimiento de la capacidad} funcional. En la población de adultos mayores que se encontraron con dependencia moderada y leve las acciones continuas de enfermería fueron enfocadas a la promoción de la salud diferenciada, en tal sentido, se organizaron los adultos en grupos según las necesidades de acompañamiento requeridas desarrollando caminatas progresivas, bailes suaves, cargar objetos, lanzamiento de pelotas de diversos tamaños y ejercicios de motricidad fina. Los grupos de adultos mayores independientes desarrollaron jornadas de movimientos amplios en el acto de bailar, trotes con supervisión y ejercicios de fortalecimiento muscular, entre otras. Las actividades físicas se realizaron con un intervalo inicial de 5 minutos acorde a la capacidad de resistencia de los participantes, para un acumulado semanal de 45 minutos aproximadamente.

El impacto de las actividades de enfermería se pudo evidenciar en el transcurso del proyecto dado que los adultos mayores demostraron más seguridad y destreza al arreglarse $(99,4 \%)$, actividad que evidenció mejor uso de la motricidad fina al abotonarse, afeitarse, subir cremallera, ponerse aretes, amarrarse los cordones; mayor capacidad de equilibrio (75\%), resistencia (82\%) y fuerza muscular (75\%) lográndose un incremento en la capacidad funcional del sistema músculo esquelético (85\%), expresado entre otros aspectos por la 
mayor resistencia al tiempo de actividad que pasó de 5 a 15 minutos por tres series para un acumulado semanal de 135 minutos.

De igual forma, manifestaron y se verificó la interiorización y el desarrollo de acciones de autocuidado tales como prácticas nutricionales adecuadas (75\%), higiene y aseo corporal (90\%), actividad física regular (70\%) y adherencia al tratamiento de sus enfermedades (78\%). En forma general se observó que los adultos mayores sujetos de esta investigación alcanzaron un mayor nivel de auto cuidado a pesar de los cambios y limitaciones propias de la senectud.

Paralelo al mejoramiento de las condiciones físicas, se pudo establecer que las actividades de enfermería aportaron a los participantes recompensas emocionales relacionadas con el mejoramiento del autoestima, automotivación y actitud positiva frente a las actividades de su vida cotidiana, dado que los participantes asumieron de forma decidida y activa su papel en el proceso, expresaron satisfacción en las visitas de seguimiento, con participación constante en los talleres y encuentros.

En lo que respecta al sistema de apoyo educativo, se realizaron visitas domiciliarias de forma continua que incluían consejerías de enfermería frente al manejo de los cambios fisiopatológicos de la etapa de la vida adulta, con miras a potenciar su capacidad de comprensión y cuidado frente a las características individuales y familiares que cada uno poseía al momento del diagnóstico. También se realizaron actividades lúdicas y relatos de evocación para estimular la capacidad mental del adulto mayor.

Lograda la atención y ejercitación de la memoria de fijación a los adultos mayores participantes se les enfatizó sobre la importancia de la participación activa en el mantenimiento de su salud y la prevención de la enfermedad, también se orientó sobre la prevención de accidentes en el hogar entre otros (Tabla 3).

La implementación de actividades de promoción y prevención en la población longeva, proporcionó a los adultos mayores y a sus familias una serie de herramientas básicas para el manejo de la salud y la enfermedad (82\%) y para aprender a mantener la funcionalidad, independencia y bienestar del adulto mayor $(87 \%)$.

Tabla 3. Actividades educativas desarrolladas en los encuentros con adultos mayores

\begin{tabular}{|c|c|}
\hline Promoción de la salud & $\begin{array}{c}\text { Prevención/control de la } \\
\text { enfermedad }\end{array}$ \\
\hline $\begin{array}{l}\text { Cambios y cuidados en la } \\
\text { senectud }\end{array}$ & $\begin{array}{l}\text { Importancia del auto cuidado en la } \\
\text { salud y en la enfermedad }\end{array}$ \\
\hline $\begin{array}{l}\text { Importancia de realizar } \\
\text { actividades para mejorar la } \\
\text { funcionalidad física y la } \\
\text { autonomía }\end{array}$ & $\begin{array}{l}\text { Prevención y cuidados en la } \\
\text { hipertensión arterial, tratamiento } \\
\text { farmacológico y no farmacológico. } \\
\text { Importancia de la adherencia al } \\
\text { tratamiento }\end{array}$ \\
\hline $\begin{array}{l}\text { Prácticas nutricionales } \\
\text { adecuadas e importancia del } \\
\text { consumo de agua hervida o } \\
\text { clorada }\end{array}$ & $\begin{array}{l}\text { Importancia y realización de: Auto } \\
\text { examen de mamas de testículos, el } \\
\text { examen de próstata y la citología }\end{array}$ \\
\hline $\begin{array}{l}\text { Importancia de la recreación } \\
\text { y utilización del tiempo libre }\end{array}$ & Prevención y cuidados en la diabetes \\
\hline $\begin{array}{l}\text { Prácticas adecuadas de aseo } \\
\text { personal e higiene de la } \\
\text { vivienda }\end{array}$ & $\begin{array}{l}\text { Prevención y manejo de problemas } \\
\text { respiratorios }\end{array}$ \\
\hline $\begin{array}{l}\text { Estrategias para contribuir a } \\
\text { mantener una buena } \\
\text { funcionalidad familiar }\end{array}$ & $\begin{array}{l}\text { Manejo de las infecciones e } \\
\text { incontinencias urinarias }\end{array}$ \\
\hline \multirow[t]{3}{*}{$\begin{array}{l}\text { Autoestima y autonomía en } \\
\text { la tercera edad }\end{array}$} & $\begin{array}{l}\text { Importancia de la prevención del } \\
\text { tabaquismo, ingesta de bebidas } \\
\text { alcohólicas }\end{array}$ \\
\hline & $\begin{array}{l}\text { Uso racional de medicamentos, } \\
\text { automedicación como problema de } \\
\text { salud }\end{array}$ \\
\hline & Prevención de accidentes en el hogar \\
\hline
\end{tabular}

b) Fortalecimiento de las capacidades de autocuidado. La prestación del cuidado de enfermería con calidad, calidez, oportuno, personalizado y con acciones participativas, permitió tras una ventana de observación de 18 meses identificar que las acciones de promoción de la salud tienen como efecto permanente al mantener al adulto mayor activo (80\%), con capacidad de regular y mejorar su nivel de autonomía (79\%) en comparación con la cifras iniciales (68\% y 62\%), la automotivación y el conocimiento adquirido le permite enfocar sus acciones a la prevención de incapacidades $(72 \%), \quad y$ asumir de forma activa 
responsabilidades en el auto cuidado de la salud y de las enfermedades propias de la senectud (82\%).

En este sentido, la mayoría de los adultos mayores (92\%) y sus cuidadores (85\%) reconocieron e interiorizaron la importancia del autocuidado y de la autonomía como elementos indispensables para tener un envejecimiento funcional, activo, agradable y saludable; dándole calidad de vida a los años.

Un resultado de gran relevancia es el derivado de las actividades recreativas y espirituales observándose en un $100 \%$ el mejoramiento en las expresiones de amistad, compañerismo, el disfrute por la vida, la libre expresión de sentimientos por los amigos y familiares, fortalecimiento de las relaciones interpersonales y excelente actitud para el trabajo en equipo con sus pares $\mathrm{u}$ homólogos, con estudiantes $\mathrm{y}$ docentes del Programa de Enfermería de la Universidad de Córdoba.

\section{Discusión}

Los resultados de este estudio se inscriben en las tendencias actuales $(9,10)$ de concebir la intervención del profesional de enfermería a través de propuestas integrales y holísticas que faciliten el análisis multidimensional de las experiencias humanas, especialmente aquellas que tienen lugar en la etapa de la vejez, donde las personas sufren transformaciones de índole biopsicosocial que ameritan abordarse desde todas las perspectivas con miras a generar mayores niveles de comprensión acerca del envejecimiento como experiencia normal del ser humano(11).

En lo que concierne al perfil sociodemográfico, los hallazgos de este estudio presentan similitudes con los resultados de otras investigaciones de la región Caribe colombiana como la de Amarís y Arrieta(12) cuando se identifica un grupo poblacional de mayorías femeninas, analfabetas, viudos, afiliados al régimen subsidiado de salud y entre las edades de 67 y 72 años, que sumados al abandono estatal, carencia de jubilación y la alta vulnerabilidad social que se produce en esta zona en particular, sitúan a los adultos mayores en grupos poblacionales frágiles, cuyas variables sociodemográficas podrían actuar como determinantes que afectan los niveles de funcionalidad y de autocuidado de la población sujeto.

En relación a la necesidad de adoptar lógicas de atención-cuidado-geriátrico diferenciales que contribuyan con el mantenimiento de la capacidad funcional del adulto mayor, es pertinente comprender que de acuerdo a estudios nacionales ${ }^{(12-16)}$ e internacionales ${ }^{(17,18)}$ esta se halla ligada a la edad y con mayor dependencia en el género femenino en condiciones de vulnerabilidad social. No obstante, en este trabajo se encontró que pese a las desventajas de las mujeres ancianas para desarrollar un estado de salud óptimo, estas poseen ventajas comparativas frente al género masculino relacionadas con niveles efectivos de participación en programas de promoción de la salud, apropiación conceptual para el autocuidado y buena disposición para adoptar hábitos saludables que les permite mejorar su capacidad funcional, constituyéndose en un factor protector que ha de incluirse en los planes de salud territoriales.

A partir de los cambios obtenidos en la población sujeto, al final de las intervenciones de enfermería, que refieren avances en el nivel de funcionalidad los cuales pueden expresarse en que $30,76 \%$ pasaron a la clasificación de independientes y 71,43\% evolucionó de la dependencia moderada a la categoría de dependencia leve, se puede decir que los adultos mayores independientes se duplicaron tras 18 meses de intervención. Si bien, estos resultados son significativamente valiosos en materia de salud, también es necesario señalar que todo proceso de intervención de enfermería amerita que se active de manera paralela a otros procesos de participación y motivación en las personas mayores como dispositivos del envejecimiento activo. No basta con generar programas de salud que busquen incrementar los niveles de la capacidad funcional del adulto sin tener en cuenta factores como la edad, que 
puede o no incrementar los niveles de participación en salud(19) los determinantes culturales que niegan la posibilidad al adulto de concebirse como un sujeto activo, independiente $\mathrm{y}$ con potencialidades que generen mayor confianza en sí mismos(20) y la identificación de redes de apoyo locales que coadyuven la realización de actividades socioeducativas que impacten en el establecimiento del diálogo de saberes y el aprendizaje dialógico(21).

Reconocer la importancia del autocuidado es esencial para el mantenimiento de la salud, porque, el autocuidado se potencia en la medida en que la persona reconoce que el mejoramiento de la salud depende de sí misma y que el contexto familiar y comunitario es un soporte significativo fundamental para que las personas se empoderen de las actividades preventivas que le permitirán mejorar su funcionalidad y lograr la independencia que todos añoran en esta etapa de la vida.

Los resultados positivos obtenidos tras la implementación del programa educativo de apoyo que se ejecutó y se sistematizó durante este estudio, obedecen al incremento de conocimientos para el autocuidado, hábitos alimentarios e importancia de la actividad física regular para la salud del geronte logros similares a los señalados por Herrera et al(22).

Es pertinente aclarar que en las experiencias que tuvieron los adultos mayores frente al desarrollo de las actividades físicas y de autocuidado se pudo evidenciar que gran parte de sus vivencias están permeadas por sus sistemas culturales(23) y el sentido que los mismos asignan a las actividades de la vida diaria como una dieta rica en harinas y carbohidratos, sedentarismo, pocas demostraciones de afecto por parte del género masculino, que obedece a una tradición patriarcal que persiste en los modelos familiares de la región, entre otras. De tal forma, que en principio se pudo identificar en algunos gerontes una resistencia para aceptar la nueva información y validar los modelos saludables para la adopción de la cultura del envejecimiento activo, lo que implicó la implementación de estrategias de persuasión, acompañamiento permanente, técnicas motivacionales y ante todo demostraciones de afecto por parte del personal de la salud que trajo consigo cambios significativos en la población intervenida.

Desde el ejercicio profesional de la enfermería se han de asumir diferentes enfoques de atención (diferenciada, derechos, género, poblacional, entre otros) que aportan desde sus sustratos teóricos conceptuales elementos importantes en la atención integral del anciano, a tal punto, que la acciones de promoción de la salud del tipo osteo-muscular, por ejemplo, coadyuvan a la transformación de las condiciones de vida para el restablecimiento de la salud del geronte cuando inciden directamente en el aumento de las capacidades funcionales en el plano biopsicosocial, o dicho de otra forma, en la medida en que se atienda al adulto mayor en el marco de sus especificidades y necesidades, se obtendrán mayores niveles de funcionalidad en el plano físico, social y psicológico.

Las actividades artísticas constituyeron una arista de gran importancia en el desarrollo de las sesiones educativas, porque a partir de estas, los adultos mayores tuvieron la oportunidad de potenciar habilidades y destrezas que favorecieron la articulación de ideas, expresar sus sentimientos con mayor facilidad y mejorar su motricidad fina. Todo este entramado de relaciones y universos de sentido que el adulto teje en torno a sí mismo se convierte en una gran oportunidad para su desarrollo integral por cuanto pasa de ser un sujeto pasivo para convertirse en agente renovador capaz de insertarse adecuada y funcionalmente en su familia y en la comunidad.

Finalmente, al momento de valorar la capacidad funcional se pudo apreciar que mediante la realización de acciones educativas con enfoques diferenciales para mantener y/o mejorar la autonomía de los longevos, se deduce que la intervención basada en la promoción de la salud potencia las capacidades del adulto mayor al máximo, toda vez que se genera un sentimiento de autovaloración que incide en la toma de decisiones frente al cuidado de su salud. Además, este punto reviste vital importancia, por 
cuanto diversos estudios(24-26) sustentan que el indicador más representativo para este grupo etario es el estado de independencia funcional y definen el estado de salud entre los envejecidos no en términos de déficit sino de mantenimiento de la capacidad funcional, que sumado a niveles máximos de satisfacción del sujeto incrementan las posibilidades de independencia y minimizan la aparición de discapacidades asociadas con el envejecimiento.

\section{Conclusiones}

Las acciones desarrolladas con enfoque promocional y de autocuidado mejoran los niveles de funcionalidad de los participantes en términos de revertir las limitaciones en la capacidad funcional: de dependencia moderada a dependencia leve y de leve a independiente, así mismo, potencializan la capacidad de regular la autosuficiencia y autonomía del sujeto beneficiando con ello la salud.

Las condiciones socio-demográficas que rodean al adulto mayor actúan como factores condicionantes que fomentan el envejecimiento activo o la incapacidad física; limitando no solo su capacidad funcional sino afectando también el mundo social y psicológico del adulto. Adicionalmente al aspecto socio-cultural que rodea al adulto mayor, este convive con el hecho de que envejecer implica reconocer una serie de pérdidas físicas, sicológicas y sociales, que acortan lentamente su proyecto de vida. Si bien es cierto, que como profesionales de salud se hace difícil transformar los cambios propios de la senectud $y$ las condiciones materiales que rodean al adulto mayor, también se puede afirmar que el acompañamiento eficaz y afectuoso incide directamente en el estado de ánimo, incrementa los niveles de motivación y mejora la participación activa y eficiente de los sujetos de cuidado.

Las acciones asistenciales de enfermería realizadas con oportunidad, calidad y calidez acompañadas simultáneamente de programas alternos de apoyo educativo en promoción de la salud, permiten mantener al adulto mayor activo, participante en grupos, con mayores niveles de conocimiento frente al autocuidado, con prácticas nutricionales adecuadas, y ejercicio físico regular; además son capaces de enfocar sus acciones en la prevención de incapacidades y de asumir responsablemente su auto cuidado tanto en la salud como en la enfermedad.

Así mismo se espera que se pueda enriquecer el criterio de pertinencia de estas áreas en el ejercicio laboral enfermero, de forma tal que este contribuya al envejecimiento funcional, saludable y digno de las personas mayores.

\section{Conflicto de intereses}

Los autores declaran que no existen conflictos de intereses.

\section{Referencias}

1. Organización Mundial de la Salud. Informe mundial sobre el envejecimiento y la salud. J Chem Inf Model. 2015;53(9):1689-99.

2. Organización Mundial de la Salud. 10 Datos Sobre El Envejecimiento y La Salud [Internet]. Ginebra: OMS; 2017. Disponible en: http://www.who.int/features/factfiles/ageing/es/

3. Ministerio de Salud y Protección Social. Envejecimiento Demográfico. Colombia 1951-2020 Dinámica Demográfica y Estructuras Poblacionales. Bogotá: MinSalud; 2013. Disponible en: https://www.minsalud.gov.co/sites/rid/Lists/Bibliote caDigital/RIDE/DE/PS/Envejecimiento-demograficoColombia-1951-2020.pdf

4. Gobernación de Córdoba. Plan de Desarrollo "Unidos por Córdoba 2016-2019" [Internet]. Córdoba: Gobernación de Córdoba; 2016. [citado 29 de junio de 2017]. Ordenanza No. 10. Disponible en: http://www.cordoba.gov.co/descargas/plan_desarroll o_2016/Plan-Desarrollo-2016-2019-UnidosCórdoba.pdf

5. Departamento Administrativo Nacional de Estadísticas. Pobreza monetaria 2015: Córdoba [Internet]. Bogotá: DANE; 2015. [citado 29 de junio de 2017]. Disponible en:

http://www.dane.gov.co/files/investigaciones/condici ones_vida/pobreza/2015/Cordoba_Pobreza_2015.pdf

6. Ministerio de Salud y Protección Social. Plan Decenal de Salud Pública, PDSP, 2012 - 2021. MinSalud: Bogotá; 2012. Disponible

http://www.minsalud.gov.co/Documentos

en: Publicaciones/Plan Decenal - Documento en consulta para aprobación.pdf

7. Aristizábal-Hoyos GP, Blanco-Borjas DM, SánchezRamos A, Ostiguín-Meléndez R. El Modelo de Promoción de la Salud de Nola Pender. Una reflexión en torno a su comprensión. Enfermería Univ ENEO-UNAM. 2011;8(4):16-23. 
8. Barrero-Solis C, Garcia-Arrioja S, Ojeda-Manzano A. Índice Barthel (IB): Un instrumento esencial para la evaluación funcional y la rehabilitación. Plasticidad y Restauración Neurológica. 2005;4:81-85.

9. Leiton Espinoza ZE. El envejecimiento saludable y el bienestar: un desafío y una oportunidad para enfermería. Enfermería Univ. 2016;13(3):139-41.

10. Urra Medina E, Hernández Cortina A. La naturaleza del conocimiento de enfermería: Reflexiones para el debate de una mejor comprensión de los cuidados. Cienc y Enferm XXI. 2015;(3):113-22.

11. Alvarado-García AM, Salazar-Maya ÁM. Análisis del concepto de envejecimiento. Gerokomos. 2014; 25(2):57-62.

12. Amarís G V, Arrieta EM. Condición sociofamiliar, asistencial y de funcionalidad del adulto mayor de 65 años en dos comunas de Sincelejo (Colombia). Salud Uninorte. 2012;28(1):75-87.

13. Pinillos-Patiño Y, Prieto-Suárez E. Funcionalidad física de personas mayores institucionalizadas $y$ no institucionalizadas en Barranquilla, Colombia. Rev salud pública. 2012;14(3):438-47.

14. Ministerio de Salud y Protección Social, Colciencias. Sabe Colombia 2015: Estudio Nacional De Salud, Bienestar Y Envejecimiento. Resumen Ejecutivo. Bogotá: Minsalud; 2015. 1-11 p. Disponible en: https://www.minsalud.gov.co/sites/rid/Lists/Bibliote caDigital/RIDE/VS/ED/GCFI/Resumen-EjecutivoEncuesta-SABE.pdf

15. Guerrero N, Yépez MC. Factores asociados a la vulnerabilidad del adulto mayor con alteraciones de salud. Rev Univ y salud. 2015;17(1):121-31.

16. Rios C, Maria C, Arango C. Medición de la inequidad en salud en adultos mayores de Medellín, 2009. Rev Fac Nac Salud Publica. 2014;32:290-304.

17. Sánchez Padilla LM, González Pérez U, Alerm González A, Bacallao Gallestey J. Calidad de vida psíquica y estado de salud física en el adulto mayor. Rev Habanera Ciencias Médicas. 2014;13(2):337-49.

18. Varela Pinedo L, Chávez Jimeno H, Tello Rodríguez T, Ortiz Saavedra P, Gálvez Cano M, Casas Vásquez $\mathrm{P}$, et al. Perfil clínico, funcional y sociofamiliar del adulto mayor de la comunidad en un distrito de Lima, Perú. Rev Peru Med Exp Salud Pública. 2015;32(4):2-9.

19. Pinillos Y, Prieto E, Herazo Y. Nivel de participación en actividades sociales y recreativas de personas mayores en Barranquilla, Colombia. Rev salud pública. 2013;15(6):850-7.

20. Miralles I. Vejez productiva: El reconocimiento de las personas mayores como un recurso indispensable en la sociedad. Kairos Rev temas Soc. 2010;(26):4.

21. Haro AE De, Margarita S, Miguel M De. La percepción de la calidad de vida en las mujeres mayores y su envejecimiento activo a través de actividades socioeducativas en los centros sociales. Rev Investig Educ. 2015;33(2):471-88.

22. Herrera $\mathrm{P}$, Martínez $\mathrm{N}$, Navarrete C. Intervención comunitaria para mejorar la calidad de vida del adulto mayor. Rev Cuba Med Integr. 2013;30(4):326-45.

23. Ramón J, Escalona J, Rodríguez R, Fernández $M$, Valdivie L, Cuello T. Intervención educativa sobre calidad de vida en el adulto mayor. Chacao. Miranda. Junio 2015. IV Jorn Cient la SOCECS Soc Cuba Educ en Ciencias la Salud Holguín. 2015:1-16.

24. Satorres-Pons E. Bienestar psicológico en la vejez y su relación con la capacidad funcional y la satisfacción vital [Tesis Doctoral]. Valencia: Universidad De Valencia; 2013. Disponible en: http://roderic.uv.es/bitstream/handle/10550/26298/ envejecimiento y bienestar.pdf?sequence $=1$

25. Monroy-Rojas A, Contreras-Garfias ME, García-Jiménez MA, García-Hernández ML, Cárdenas-Becerril L, RiveroRodríguez LF. Estatus funcional de adultos mayores de Tláhuac, Ciudad de México. Enfermería Universitaria. 2016;13(1):25-30.

26. Bejines M, Velasco R, Garcia L, Barajas A, Aguilar L, Rodriguez M. Valoración de la capacidad funcional del adulto mayor residente en casa hogar. Rev Enferm del Inst Mex Segur Soc. 2015;23(1):9-15. 